\title{
The intrinsic asymmetry and inhomogeneity of Teichmüller space
}

\author{
Benson Farb and Shmuel Weinberger *
}

July 27, 2021

\section{Introduction}

Let $S=S_{g, n}$ be a connected, orientable surface of genus $g \geq 0$ with $n \geq 0$ punctures. Let Teich $(S)$ denote the corresponding Teichmüller space, and let $\operatorname{Mod}(S)$ denote the mapping class group of $S$. Understanding the analogy of Teich $(S)$ with symmetric spaces is a wellknown theme. Recall that a complete Riemannian manifold $X$ is symmetric if it is symmetric at each point $x \in X$ : the map $\gamma(t) \mapsto \gamma(-t)$ which flips geodesics about $x$ is an isometry. Symmetric spaces $X$ are homogeneous: the isometry group $\operatorname{Isom}(X)$ acts transitively on $X$. In his famous paper [Ro, Royden studied the possible symmetry and homogeneity of Teich $(S)$, endowed with the Teichmüller metric.

Theorem 1.1 (Royden [Ro]). Suppose $S$ is closed of genus at least 2, and let Teich $(S)$ be Teichmüller space endowed with the Teichmüller metric $d_{\text {Teich. }}$. Then

a. $\left(\right.$ Teich $\left.(S), d_{\text {Teich }}\right)$ is not symmetric at any point.

b. Isom(Teich $\left.(S), d_{\text {Teich }}\right)$ contains $\operatorname{Mod}(S)$ (modulo its center if $S$ is closed of genus 2) as a subgroup of index 2 .

Note that the conclusion of Theorem 1.1 is false when $\operatorname{genus}(S)=1$, as $d_{\text {Teich }}$ in this case is the hyperbolic metric on the upper half-plane. Earle-Kra [EK] extended part (b) of Royden's Theorem to arbitrary surfaces of finite type. Royden deduced Theorem 1.1 from a detailed analysis of the fine structure of the space $\mathrm{QD}^{1}(M)$ of unit norm holomorphic quadratic differentials on a Riemann surface $M$. In particular, he found an embedding of $M$ in $\mathrm{QD}^{1}(M)$ and characterized it by the degree of Holder regularity of the norm on $\mathrm{QD}^{1}(M)$ at the points of the embedding.

The Teichmüller metric is a complete Finsler metric, under which moduli space $\mathcal{M}(S):=$ $\operatorname{Teich}(S) / \operatorname{Mod}(S)$ has finite volume (see the proof of Theorem 8.1 of [Mc] 1$]$. There are many other complete, finite volume, $\operatorname{Mod}(S)$-invariant Finsler (indeed Riemannian) metrics on Teich $(S)$, each with special properties. Examples include the Kahler-Einstein metric,

\footnotetext{
*Both authors are supported in part by the NSF.

${ }^{1} \mathrm{~A}$ Finsler metric determines a unique volume form by declaring the unit Finsler ball at each point to have volume 1 .
} 
McMullen's metric, and the (perturbed) Ricci metric. Following Royden's approach to understanding the symmetry and homogeneity of these metrics appears to involve difficult analysis.

The goal of this paper is to explain a completely different, nonanalytic mechanism behind Royden's Theorem. It will allow us to extend much of his result from the Teichmüller metric to any metric, including each of those mentioned above. The theorem is the following.

Theorem 1.2. Let $S=S_{g, n}$ be a surface, and let $d$ be any complete, finite covolume, $\operatorname{Mod}(S)$-invariant Finsler (e.g. Riemannian) metric on Teich $(S)$. Then

a. If $g \geq 3$ then $($ Teich $(S), d)$ is not symmetric at any point.

b. If $3 g-3+n \geq 2$ then $\operatorname{Isom}(\operatorname{Teich}(S), d)$ contains $\operatorname{Mod}(S)$ (modulo its center if $(g, n)=$ $(1,2)$ or $(2,0))$ as a subgroup of finite index.

In this way Teichmüller space exhibits a kind of intrinsic asymmetry and inhomogeneity. The number $3 g-3+n$ plays the role of "Q-rank" in this context. Theorem $1.2(\mathrm{~b})$ is sharp: the conclusion is false whenever $3 g-3+n<2$, and indeed the corresponding Teichmüller spaces admit hyperbolic metrics.

\section{Remarks.}

1. The proof of Theorem 1.2 gives immediately that the hypothesis can be weakened to allow $d$ to be any $\Gamma$-invariant metric, for any finite index subgroup $\Gamma<\operatorname{Mod}(S)$. As such subgroups are typically torsion free, this shows that the phenomenon of inhomogeneity and asymmetry is not the result of the constraints imposed by having finite order symmetries of the metric.

2. Ivanov proved (see, e.g., [I2]) that the moduli space of Riemann surfaces Teich $(S) / \operatorname{Mod}(S)$ never admits a locally symmetric metric when $S$ is closed and $\operatorname{genus}(S) \geq 2$. If it did admit such a metric, then $\operatorname{Teich}(S)$ would admit a complete, finite covolume, $\operatorname{Mod}(S)$ invariant metric which is symmetric at every point. Thus Theorem 1.2(a) gives a new proof, and generalization, of Ivanov's theorem.

The proof of Theorem 1.2(a) relies on Theorem 1.2(b). One key ingredient in our proofs is Smith theory. This is not the first time Smith theory has been used to analyze actions on Teichmüller space: Fenchel used it in the 1940's to analyze certain periodic mapping classes.

We conjecture that the index in Theorem 1.2(b) can be taken to depend only on $S$. More strongly, one might hope that it can always be taken to be 2. As evidence towards this strongest possible conjecture, we can prove it in the "Q-rank 2" case.

Theorem 1.3. Let $S$ be the twice-punctured torus or the 5-punctured sphere. Then the index in Theorem 1.2(b) can be taken to be 2 .

Acknowledgements. We are grateful to Tom Church, Alex Eskin and Dan Margalit for their numerous useful and insightful comments. We are especially grateful to Yair Minsky, whose persistence in pinning us down, and whose many comments and corrections, greatly improved this paper. 


\section{Proof of Theorem 1.2(b)}

By the Myers-Steenrod Theorem (or, in the general Finsler case, Deng-Hou [DH]), the group $I:=\operatorname{Isom}(\operatorname{Teich}(S), d)$ is a Lie group, possibly with infinitely many components, acting properly discontinuously on Teich $(S)$. We remark that Theorem 2.2 of [DH] states that any isometry of a Finsler metric is necessarily a diffeomorphism; we will use this smoothness later.

Let $I_{0}$ denote the connected component of the identity of $I$; note that $I_{0}$ is normal in $I$. Let $\mu$ denote the measure on $\operatorname{Teich}(S)$ (and the induced measure on $\operatorname{Teich}(S) / \operatorname{Mod}(S)$ ) induced by the Finsler metric $d$. We are assuming that $\mu(\operatorname{Teich}(S) / \operatorname{Mod}(S))<\infty$.

If $I$ is discrete, then we claim that $[I: \operatorname{Mod}(S)]<\infty$. To see this, let $K_{I}$ be a measuretheoretic fundamental domain for the $I$-action on Teich $(S)$. By this we mean that:

1. The complement of $\bigcup_{g \in I} g \cdot K_{I}$ in Teich $(S)$ has $\mu$-measure 0 , and

2. For all nontrivial $g \in I$, we have $\mu\left(g \cdot K_{I} \cap K_{I}\right)=0$.

Note that measurable fundamental domains always exist for properly discontinuous actions. Let $K_{\operatorname{Mod}(S)}$ be a measure-theoretic fundamental domain for $\operatorname{Mod}(S)$. Let $\left\{g_{i}\right\}$ be a collection of coset representatives for $\operatorname{Mod}(S)$ in $I$. Then we can choose $K_{\operatorname{Mod}(S)}$ so that

$$
K_{\operatorname{Mod}(S)}=\bigcup_{i} g_{i} \cdot K_{I}
$$

Notice that this union is a disjoint union, up to sets of measure zero, by Condition (2) in the definition of fundamental domain. Since by assumption $\mu\left(K_{I}\right)$ and $\mu\left(K_{\operatorname{Mod}(S)}\right)$ are both finite, it follows that this must be a finite union, i.e. $[I: \operatorname{Mod}(S)]<\infty$.

So suppose that $I$ is not discrete. Myers-Steenrod then gives that the dimension of $I$ is positive, and so $I_{0}$ is a connected, positive-dimensional Lie group. Let $\Gamma:=\operatorname{Mod}(S)$, and let $\Gamma_{0}:=I_{0} \cap \operatorname{Mod}(S)$. We have the following exact sequences:

$$
1 \rightarrow I_{0} \rightarrow I \rightarrow I / I_{0} \rightarrow 1
$$

and

$$
1 \rightarrow \Gamma_{0} \rightarrow \Gamma \rightarrow \Gamma / \Gamma_{0} \rightarrow 1
$$

Step 1 ( $\Gamma_{0}$ is a lattice in $\left.I_{0}\right)$ : We begin by replacing $\Gamma$ (hence $\Gamma_{0}$ ) by a torsion-free subgroup of finite index, but keep the notation $\Gamma$ (resp. $\Gamma_{0}$ ) for this new group. While not formally necessary, this assumption will make "orbifold technicalities" disappear; the point is that quotients of Teich $(S)$ and associated bundles by $\Gamma$ will be manifolds.

We begin by replacing the proper action of $I$ on Teich $(S)$ by a free action on an associated space $\mathcal{F}(\operatorname{Teich}(S))$, which we now describe.

For any smooth, connected, $n$-dimensional Finsler manifold $M$ we have the natural unit sphere-bundle over $M$, which is a sub-bundle of the tangent bundle $T M$, with fiber over $m \in M$ the unit Finsler sphere $S_{m}$ of $T M_{m}$. We also have the associated bundle $E \rightarrow M$ whose fiber is the $n$-fold product $S_{m}^{n}$. Let $\mathcal{F}(M)$ denote the sub-bundle of this bundle with 
fiber the set of $n$-tuples of distinct points of $S_{m}$ that $\operatorname{span} T M_{m}$. The group $\operatorname{Isom}(M)$ clearly acts on $\mathcal{F}(M)$ by homeomorphisms.

The exponential map on a Finsler manifold is a local diffeomorphism (see, e.g. [DH], Lemma 1.1). Since we also have that Finsler isometries take geodesic rays to geodesic rays, we see that the set of points of $M$ for which a Finsler isometry is the identity and has derivative the identity is both open and closed. Thus the action of $\operatorname{Isom}(M)$ on $\mathcal{F}(M)$ is free.

Now, we will want to apply the Slice Theorem to this action (see below), and to do so we need it to preserve some smooth structure on $\mathcal{F}(M)$. To this end, first note that $M$ is assumed to be a smooth manifold, and since $\operatorname{Isom}(M)$ acts properly on $M$, we have that $\operatorname{Isom}(M)$ actually preserves some Riemannian metric on $M$. We then note that there is an $\operatorname{Isom}(M)$-equivariant homeomorphism from $\mathcal{F}(M)$ to the bundle of unit $n$-frames over $M$, and so the action of $\operatorname{Isom}(M)$ on $\mathcal{F}(M)$ preserves the pullback of the standard smooth structure on the latter bundle. We remark that the reason we do not simply replace the given Finsler metric with this invariant Riemannian metric is that the finiteness of volume of $M$ in the Finsler metric need not imply such finiteness for the invariant Riemannian metric.

We now wish to construct an $\operatorname{Isom}(M)$-invariant measure on $\mathcal{F}(M)$. To this end, note that the bundle $E \rightarrow M$ discussed above is locally a product $U \times S^{n}$, where $U$ is a neighborhood in $M$. The Finsler metric on $M$ determines a volume form on $M$, which induces a measure $\nu$ on $U$. On $S$, we have an induced measure $\mu$ which is given infinitessimally by the rule that, for a subset $A \subseteq S_{m}$, the measure is given by the measure (induced by the Finsler norm of $A$ on $T M_{m}$ ) of the Euclidean cone of $A$, normalized so that the measure of $S_{m}$ equals 1 . The local product measure $\nu \times \mu$ then gives an $\operatorname{Isom}(M)$-invariant measure on $E$, which in turn induces an $\operatorname{Isom}(M)$-invariant measure on $\mathcal{F}(M)$. By construction, the pushforward of this measure under the natural projection $\mathcal{F}(M) \rightarrow M$ is the measure on $M$ induced by the given Finsler metric; in particular, if $M$ is assumed to have finite measure then $\mathcal{F}(M)$ has finite measure.

Now let $M=\operatorname{Teich}(S)$. Pick $x \in \mathcal{F}(M)$, and consider the $I$-orbit $O_{x}$. The Slice Theorem for proper group actions (see, e.g., [DK] 2.4.1) in this context asserts that there is an $I$-invariant tubular neighborhood $V$ of $O_{x}$ in $\mathcal{F}($ Teich $(S))$ that is a homogeneous vector bundle $\pi: V \rightarrow O_{x}$. The measure on $\mathcal{F}(M)$ constructed above restricts to a measure on $V$, and $\pi$ pushes this forward to a left-invariant measure on $O_{x}$, which we can identify with a left-invariant measure on $I$. Note that all left-invariant measures on $I$ are proportional, by uniqueness of Haar measure. The key property we will use is that if a subset $A \subseteq I$ has infinite measure then $\pi^{-1}(A)$ has infinite measure.

Choose any fiber $D$ of the bundle $V \rightarrow O_{x}$, so that $V$ is the $I$-orbit of $D$. Since $I_{0}$ is the connected component of the identity of $I$, and since $I_{0}$ is a closed subgroup of $I$, we have that $V$ can be written as a disjoint union of $I_{0}$-orbits of $D$, one for each element of $\pi_{0}(I)$. Note that $\Gamma / \Gamma_{0} \subseteq \pi_{0}(I)$. Thus $V / \Gamma$ is given by the image of the $I_{0}$-orbit $W$ of $D$ under the projection

$$
\mathcal{F}(\operatorname{Teich}(S)) \rightarrow \mathcal{F}(\operatorname{Teich}(S)) / \Gamma=(\operatorname{Teich}(S) / \Gamma)=\mathcal{F}(\mathcal{M}(S)) .
$$

Since the $I$-action on $\mathcal{F}($ Teich $(S))$ is free, this projection is a measure-preserving homeomorphism when restricted to $W$. Now if $I_{0} / \Gamma_{0}$ had infinite measure, then so would $W$ (by the discussion above), and thus so would $\mathcal{F}(\mathcal{M}(S))$. On the other hand the map 
$\mathcal{F}(\mathcal{M}(S)) \rightarrow \mathcal{M}(S)$ is measure-preserving by the construction of the measure on $\mathcal{F}(\mathcal{M}(S))$ (see above). This gives that $\mathcal{M}(S)$ has infinite measure, contradicting the given. We conclude that $I_{0} / \Gamma_{0}$ has finite measure, as desired.

Step 2 ( $I_{0}$ is semisimple with finite center): For any connected Lie group $G$ there is an exact sequence

$$
1 \rightarrow G^{\mathrm{sol}} \rightarrow G \rightarrow G^{\mathrm{ss}} \rightarrow 1
$$

where $G^{\text {sol }}$ denotes the solvable radical of $G$ (i.e. the maximal connected, normal, solvable Lie subgroup of $G$ ), and where $G^{\text {ss }}$ is the connected semisimple Lie group $G / G^{\text {sol }}$.

We can apply this setup with $G=I_{0}$. We now follow exactly the argument of the proof of Proposition 3.3 of [FW], with the only change being that here $\Gamma$ is not assumed torsion free. First, a theorem of Raghunathan gives a unique maximal normal solvable subgroup $\Gamma_{0}^{\text {sol }}$ of $\Gamma_{0}$. Since $\Gamma_{0}^{\text {sol }}$ is unique it is characteristic in $\Gamma_{0}$, hence normal in $\Gamma$. The main theorem of [BLM] states that any solvable subgroup of $\Gamma=\operatorname{Mod}(S)$ has an abelian subgroup of finite index. Hence $\Gamma_{0}^{\text {sol }}$ has a torsion-free, normal (in $\Gamma$ ) finite-index abelian subgroup, which we denote by $N$. We will prove below that $\Gamma=\operatorname{Mod}(S)$ has no infinite normal abelian subgroups, from which we will conclude that $N$ is trivial, so that $\Gamma_{0}^{\text {sol }}$ is finite.

Now the next part of the argument of Proposition 3.3 of [FW] quotes a theorem of Prasad. In applying Prasad's theorem, we used the fact that the sum of the ranks of the abelian quotients of the derived series of $\Gamma_{0}^{\text {sol }}$ equals 0 . While in [FW this followed from the fact that $\Gamma_{0}^{\text {sol }}=0$, we only need that $\Gamma_{0}^{\text {sol }}$ is finite, which we have just proven. We may thus quote Prasad's theorem and conclude, as in [FW], that $I_{0}^{\text {sol }}$ is compact, and that the center $Z\left(I_{0}\right)$ is finite.

Given this, we finish the proof of Step 2 as follows. Since any compact Lie group is the product of a simple Lie group and a torus, $I_{0}^{\text {sol }}$ must be a torus. Further, as is precisely argued in Proposition 3.3 of [FW], the conjugation action of the connected group $I_{0}^{\text {ss }}$ on the torus $I_{0}^{\text {sol }}$, which has discrete automorphism group, must be trivial, so that $I_{0}^{\text {sol }}$ must be a direct factor of $I_{0}$. We thus need to rule this out when $I_{0}^{\text {sol }}$ is positive-dimensional.

So if we can prove that $\operatorname{Mod}(S)$ has no infinite, normal abelian subgroup $A$, and if we can rule out that $I_{0}^{\text {sol }}$ is a torus direct factor of $I_{0}$, then we have completed Step 2. We begin with the first claim.

By the classification of abelian subgroups of $\operatorname{Mod}(S)$ (see [BLM] or [I1]), any abelian subgroup $A$, after perhaps being replaced by a finite index characteristic subgroup if necessary, either is cyclic with a pseudo-Anosov generator or there is a unique maximal finite collection $C$ of simple closed curves, called the canonical reduction system of $A$, left invariant (setwise) by each $a \in A$. In the first case, the normalizer of $A$ is virtually cyclic, a contradiction, so suppose we are in the latter case. Then for any $f \in \operatorname{Mod}(S)$ the canonical reduction system for $f A f^{-1}$ is $f(C)$. The result now follows by picking an $f$ such that $f(C) \neq C$. Thus $A$ must be trivial.

We now rule out that $I_{0}$ contains a positive-dimensional torus as a direct factor. Suppose it does. Then $Z\left(I_{0}\right)$ is positive-dimensional. Since $Z\left(I_{0}\right)$ is a positive-dimensional abelian Lie group, and we can write $Z\left(I_{0}\right)=A \times\left(S^{1}\right)^{d} \times \mathbf{R}^{k}$ for some $d>0, k \geq 0$. Now let $T$ denote the maximal compact subgroup of the connected component of the identity of $Z\left(I_{0}\right)$. From the above description of $Z\left(I_{0}\right)$, it is clear that $T$ is characteristic in $Z\left(I_{0}\right)$, hence in $I_{0}$. Since $I_{0}$ is normal in $I$, we have that the conjugation action of $\Gamma=\operatorname{Mod}(S)$ leaves 
$T$ invariant. Thus the action of $\Gamma$ on $\operatorname{Teich}(S)$ leaves $\operatorname{Fix}(T)$ invariant. Since $T$ is acting smoothly, $\operatorname{Fix}(T)$ is a manifold. Since Teich $(S)$ is contractible, $\operatorname{Fix}(T)$ is acyclic (see, e.g., $\mathrm{Br}$, Theorem 10.3). As $T$ is positive dimensional and connected, and since the $T$-action on $\operatorname{Teich}(S)$ is faithful, $\operatorname{dim}(\operatorname{Fix}(T))<\operatorname{dim}(\operatorname{Teich}(S))-1$. Note that the action of $\operatorname{Mod}(S)$ on $\operatorname{Fix}(T)$ is properly discontinuous, being the restriction of the properly discontinuous action of $\operatorname{Mod}(S)$ on $\operatorname{Teich}(S)$.

Case A $(\operatorname{dim}(\operatorname{Fix}(T))>3)$ : We claim that there is a contractible manifold $Z$ of dimension $\operatorname{dim}(\operatorname{Fix}(T))+1<\operatorname{dim}(\operatorname{Teich}(S))$ on which $\operatorname{Mod}(S)$ acts properly discontinuously. Given this, we recall that Despotovic [D] proved that $\operatorname{Mod}(S)$ admits no properly discontinuous action on any contractible manifold of dimension $<\operatorname{dim}(\operatorname{Teich}(S)$ ), giving us a contradiction. Thus $T$ would be trivial, and so $I_{0}$ is semisimple with finite center.

We now prove the claim. Note that by Smith theory (see $[\mathrm{Br}]) \operatorname{Fix}(T)$ is acyclic. We want to replace this $\operatorname{Mod}(S)$-manifold by another one that is contractible, i.e. we want to kill the fundamental group. If $\operatorname{Fix}(T) / \operatorname{Mod}(S)$ is compact, then the kernel that we are trying to kill is finitely normally generated, and the construction is standard: one kills the elements of this kernel by surgering the circles, giving rise to new homology = homotopy in dimension 2, which can then be killed by surgering the 2-spheres as well (see Section 3 of $[\mathrm{H}])$. Taking the product of $\operatorname{Fix}(T)$ with $\mathbf{R}$ if $\operatorname{dim}(\operatorname{Fix}(T))=4$, the 2 -spheres needed at this stage can be embedded by general position since $\operatorname{dim}(\operatorname{Fix}(T) \times \mathbf{R})>4$.

If $\operatorname{Fix}(T) / \operatorname{Mod}(S)$ is not compact, then one wants to do the same argument, but one has to be careful to make sure that the circles and 2-spheres that one wants to surger do not accumulate. However, by replacing $\operatorname{Fix}(T)$ by $\operatorname{Fix}(T) \times \mathbf{R}$, this problem disappears: one simply does the $i^{\text {th }}$ surgery at the "height" $\mathrm{Fix}(T) \times\{i\}$, producing at height i a 2-dimensional homology class. This class can be represented by a sphere which lies in a compact region that is above level $i-1 / 2$ (by the Hurewicz theorem) that is embedded (by general position) with trivial normal bundle (by appropriate framing). The infinite collection of constructed 2 -spheres do not accumulate, since at most $i$ of them pass through level $i$. Surgering this free basis for the homology of the previous stage gives us back an acyclic manifold, which is now, in addition, simply connected, and, thus, contractible.

Case B $(\operatorname{dim}(\operatorname{Fix}(T)) \leq 3)$ : In this case the proper action of $\operatorname{Mod}(S)$ on the acyclic space $\operatorname{Fix}(T)$ implies that the virtual cohomological dimension $\operatorname{vcd}(\operatorname{Mod}(S))$ satisfies

$$
\operatorname{vcd}(\operatorname{Mod}(S)) \leq \operatorname{dim}(\operatorname{Fix}(T)) \leq 3 .
$$

But then by the formulas for $\operatorname{vcd}(\operatorname{Mod}(S))$, given for example in Theorem 6.4 of [I2], and since $3 g-3+n \geq 2$ by hypothesis, this leaves the cases of possible $(g, n)$ to be one of $\{(2,0),(0,5),(0,6),(1,2),(1,3)\}$. As the action of $\operatorname{Mod}(S)$ on $\operatorname{Fix}(T)$ is properly discontinuous, this rules out $\operatorname{dim}(\operatorname{Fix}(T))=0$. Now $\operatorname{Fix}(T)$ has even codimension in $\operatorname{Teich}(S)$ (see $[\mathrm{Br}]$, Theorem 10.3), and so is even-dimensional. As we are assuming in this case that $\operatorname{dim}(\operatorname{Fix}(T)) \leq 3$, it follows that $\operatorname{dim}(\operatorname{Fix}(T))=2$. But this would imply that $\operatorname{Mod}(S)$ has a a (closed or open) surface group as a subgroup of finite index. It cannot, however, because for instance in these cases $\operatorname{Mod}(S)$ contains both $\mathbf{Z}^{2}$, eliminating all surfaces but the torus, and also a rank 2 free group, eliminating the torus. 
Step 3 ( $I_{0}$ has no compact factors): Let $K$ be the maximal compact factor of $I_{0}$. Since $I_{0}$ is semisimple with finite center, $K$ is characteristic in $I_{0}$. Since $I_{0}$ is normal in $I$, it follows that $K$ is invariant under conjugation by any element of $I$. Further, note that $K$ is semisimple since $I_{0}$ is semisimple.

Since $K$ is compact, we have that $K \cap \Gamma=K \cap \Gamma_{0}$ is a finite normal subgroup of $\Gamma$. Replacing $\Gamma$ by a finite index subgroup, which we will also denote by $\Gamma$, we can assume that $K \cap \Gamma$ is trivial. As $K$ is invariant by conjugation by elements of $I$, we have an exact sequence

$$
1 \rightarrow K \rightarrow\langle K, \Gamma\rangle \rightarrow \Gamma \rightarrow 1
$$

where the middle term denotes the subgroup of $I$ generated by $K$ and $\Gamma$. As explained in IV.6 of [Bro], any exact sequence

$$
1 \rightarrow A \rightarrow B \rightarrow C \rightarrow 1
$$

is determined by two pieces of data: a representation $\rho: C \rightarrow \operatorname{Out}(A)$ and a cocycle $\eta \in H^{2}(C, Z(A))$, where $Z(A)$ denotes the center of $A$, and is a $C$-module via the action of $\rho$. In the case (4), we have that both $Z(K)$ and $\operatorname{Out}(K)$ are finite since $K$ is semisimple. We may thus pass to a finite index subgroup $\Lambda<\Gamma$ so that $\rho$ has trivial image. Now let

$$
1 \rightarrow Z(K) \rightarrow \widehat{\Lambda} \rightarrow \Lambda \rightarrow 1
$$

be the group extension corresponding to the pullback of the cocycle $\eta \in H^{2}(\Lambda, Z(K))$ corresponding to the extension (4) restricted to $\Lambda$. Note that this exact sequence defines a trivial cocycle. Note also that $\widehat{\Lambda} \subseteq\langle K, \Lambda\rangle$. Now $Z(K)$ is central both in $\widehat{\Lambda}$ and in $K$. We thus have a split exact sequence

$$
1 \rightarrow K / Z(K) \rightarrow\langle K, \widehat{\Lambda}\rangle \rightarrow \widehat{\Lambda} \rightarrow 1
$$

Since $\rho$ has trivial image, we can change the section of (55) to get a copy of $K / Z(K) \times \widehat{\Lambda}$ in $\langle K, \widehat{\Lambda}\rangle$. As noted above, this group is a subgroup of $\langle K, \Gamma\rangle$, and so acts on Teich $(S)$.

If $K$ is positive-dimensional then so is $K / Z(K)$, and so $K / Z(K)$ contains a closed subgroup isomorphic to a circle $T$. As the (possibly noneffective) action of $\widehat{\Lambda}$ on $\operatorname{Teich}(S)$ commutes with the action of $T$, we have that $\widehat{\Lambda}$ leaves $\operatorname{Fix}(T)$ invariant. But this gives a contradiction, exactly as in Step 2 above, once we observe that [D] applies to $\widehat{\Lambda}$, and so we obtain that $K$ is trivial.

To see that [D] applies to $\widehat{\Lambda}$, there are two minor issues: her result is stated for $\Gamma=$ $\operatorname{Mod}(S)$, while we need the theorem for finite extensions and finite index subgroups of $\Gamma$. For the first issue we simply note that, just as mentioned in the first sentence of the proof of Theorem 26 in [BKK], the groups for which the theorem in [D] holds are closed under finite extensions. The proof that $[\mathrm{D}]$ holds not just for $\operatorname{Mod}(S)$, but for any finite index subgroup $\Gamma^{\prime}$ of $\operatorname{Mod}(S)$, is verbatim the same as in [D], replacing the "Mess subgroups" $B_{g}$ constructed there with $B_{g} \cap \Gamma^{\prime}$, which has finite index in $B_{g}$. The two key properties of $B_{g}$ are:

- $B_{g}$ is the fundamental group of a closed, triangulable topological manifold of dimension $4 g-5$, and 
- The natural "point pushing" subgroup of $B_{g}$ is a closed surface group.

Each of these properties is clearly preserved by taking finite index subgroups, and so the proof of the main theorem of $[\mathrm{D}]$ goes through when $\operatorname{Mod}(S)$ replaced by any finite index subgroup $\Gamma^{\prime}$.

Step 4 ( $I_{0}$ is trivial): By the previous steps, we know that $\Gamma_{0}$ is a lattice in the semisimple Lie group $I_{0}$, and that $I_{0}$ has no compact factors. The proof of Proposition 3.1 of [FW] now gives that there is a finite index subgroup $\Gamma^{\prime}$ of $\Gamma$ so that

$$
\Gamma^{\prime} \approx \Gamma_{0} \times \Gamma^{\prime} / \Gamma_{0}
$$

To give an idea of the proof of (6) from [FW], we begin by considering the exact sequence

$$
1 \rightarrow \Gamma_{0} \rightarrow \Gamma \rightarrow \Gamma / \Gamma_{0} \rightarrow 1
$$

As mentioned above, the extension, (77) is determined by a representation $\rho: \Gamma / \Gamma_{0} \rightarrow$ $\operatorname{Out}\left(\Gamma_{0}\right)$, and by a cohomology class in $H^{2}\left(\Gamma / \Gamma_{0}, Z\left(\Gamma_{0}\right)_{\rho}\right)$.

One shows that the image of $\rho$ actually lies in $\operatorname{Out}\left(I_{0}\right)$, which is finite since $I_{0}$ is semisimple with finite center. After replacing $\Gamma$ by a finite index subgroup $\Gamma^{\prime}$, one then gets that the resulting representation $\rho$ is trivial. One can also choose $\Gamma^{\prime}$ so that $\Gamma_{0} \cap \Gamma^{\prime}$ is torsion-free. Since this group is a lattice in the semisimple Lie group with finite center $I_{0}$, it has finite center; since $\Gamma_{0} \cap \Gamma^{\prime}$ is torsion-free, its center is trivial, so that the pertinent $H^{2}$ vanishes.

We now claim that for any finite index subgroup $\Gamma^{\prime}<\operatorname{Mod}(S)$, if $\Gamma^{\prime}=A \times B$ then either $A$ or $B$ is finite. To see this, note that any such $\Gamma^{\prime}$ contains a pseudo-Anosov homeomorphism $f$ (for example take a sufficiently high power of any pseudo-Anosov in $\operatorname{Mod}(S)$ ). The centralizer in $\Gamma^{\prime}$ (indeed in $\operatorname{Mod}(S)$ ) of any power of $f$ has $\mathbf{Z}$ as a finite index subgroup (see [1], Lemma 8.13). But in a product of two infinite groups, it is easy to see that any element has some power whose centralizer does not contain $\mathbf{Z}$ as a finite index subgroup.

Thus either $\Gamma_{0}$ is finite or $\Gamma^{\prime} / \Gamma_{0}$ is finite. The latter possibility implies that $\Gamma^{\prime}$, hence $\operatorname{Mod}(S)$, has a finite index subgroup which is isomorphic to a lattice in the semisimple Lie group $I_{0}$. If $I_{0}$ is nontrivial, then it must contain a noncompact factor (by Step 3). This would then contradict the theorem of Ivanov (see, e.g., $\S 9.2$ of [I2] that no finite index subgroup of $\operatorname{Mod}(S)$ is isomorphic to a lattice in a noncompact semisimple Lie group. Thus it must be that either $I_{0}$ is trivial, or $\Gamma_{0}$ is finite. If the latter possibility were to occur, then $I_{0}$ would be compact since $\Gamma_{0}$ is a lattice in $I_{0}$ by Step 1 . But this would contradict Step 3 .

$\diamond$

\section{Proof of Theorem $1.2(\mathrm{a})$}

Let $\tau$ be a symmetry of (Teich $(S), d)$, i.e. an isometric involution with an isolated fixed point. Let $L=\langle\operatorname{Mod}(S), \tau\rangle$ be the group generated by $\operatorname{Mod}(S)$ and by $\tau$.

By Theorem 1.2 (b), which we have already proven, $[L: \operatorname{Mod}(S)]<\infty$. Thus the action of $\tau$ on $L$ by conjugation induces a commensuration of $\operatorname{Mod}(S)$, i.e. an isomorphism between two finite index subgroups. Since $\operatorname{Mod}(S)$ is residually finite, we can pass to further finite 
index subgroups so that neither contains the hyperelliptic involution. By a theorem of Ivanov (see Theorem 8.5A of [I2]), since $\operatorname{genus}(S) \geq 2$ any such commensuration agrees on some finite index characteristic subgroup $H$ of $\operatorname{Mod}(S)$ with conjugation by some element $\phi$ of the extended mapping class group $\operatorname{Mod}^{ \pm}(S)$, the index 2 supergroup of $\operatorname{Mod}(S)$ which includes an orientation-reversing homotopy class of homeomorphism.

We now claim that there exists an infinite order element $\psi_{2} \in \operatorname{Mod}(S)$ that commutes with $\tau$. Note that since the conjugation action of $\tau$ on $H$ agrees with the conjugation action of $\phi$, it is enough to produce an infinite order element $\psi_{2} \in H$ so that $\psi_{2}$ commutes with $\phi$.

Given this claim, we complete the proof of the theorem as follows. We are given that $\tau$ has an isolated fixed point $x \in \operatorname{Teich}(S)$. By Smith theory, $\operatorname{Fix}(\tau)$ is $\mathbf{Z} / 2 \mathbf{Z}$ acyclic; in particular $\operatorname{Fix}(\tau)$ is connected. Since $\psi_{2}$ is an infinite order mapping class, we have that $\psi_{2}(x) \neq x$, by proper discontinuity of the action of $\operatorname{Mod}(S)$ on $\operatorname{Teich}(S)$. But

$$
\tau\left(\psi_{2}(x)\right)=\psi_{2}(\tau(x))=\psi_{2}(x)
$$

so that $\tau$ also fixes $\psi_{2}(x) \neq x$. As $\operatorname{Fix}(\tau)$ is connected and fixes at least two distinct points, it must have positive dimension. This contradicts the fact that $x$ is an isolated fixed point of $\tau$. Thus such a $\tau$ cannot exist, and we are done.

We now prove the claim. First note that since $\tau^{2}=\mathrm{Id}$, conjugation by $\phi^{2}$ is the identity on some finite index subgroup $H$ of $\operatorname{Mod}(S)$. Now there exists $N>0$ so that for a Dehn twist $T_{\alpha}$ about any simple closed curve $\alpha$, we have $T_{\alpha}^{N} \in H$. For any twist $T_{\alpha}$ and any element $f \in \operatorname{Mod}(S)$, we have the well-known formula

$$
f T_{\alpha}^{N} f^{-1}=T_{f(\alpha)}^{N}
$$

Since $\phi^{2} \in \operatorname{Mod}(S)$, we can apply this formula with $f=\phi^{2}$, giving that $T_{\alpha}^{N}=T_{\phi^{2}(\alpha)}^{N}$ for all simple closed curves $\alpha$. Since any positive power of a Dehn twist about a curve determines that curve, we have that $\phi^{2}(\alpha)=\alpha$ for each $\alpha$. It follows that either $\phi^{2}=\operatorname{Id}$ or $\operatorname{genus}(S)=2$ and $\phi^{2}$ is the hyperelliptic involution; our assumption that $\operatorname{genus}(S)>2$ rules out the second possibility, so that $\phi=\mathrm{Id}$, and so commutes with any element $\psi_{2} \in \operatorname{Mod}(S)$. We then pick $\psi_{2} \in H$ to have infinite order. So we can assume $\phi^{2}=\mathrm{Id}$ and $\phi \neq \mathrm{Id}$.

Now any element $\phi \in \operatorname{Mod}^{ \pm}(S)$ of order 2 is represented by a homeomorphism $\phi$ of order 2 (by a theorem of Fenchel). We now assume that $g=\operatorname{genus}(S)>2$. First suppose that $\operatorname{Fix}(\phi)$ is discrete. Then $S$ two-fold branched covers $S /\langle\phi\rangle$. Since $g=\operatorname{genus}(S) \geq 3$, the Riemann-Hurwitz formula easily implies that either genus $(S /\langle\phi\rangle)>0$ or that there are at least 4 branch points on $S /\langle\phi\rangle$. Either way, the quotient $S /\langle\phi\rangle$ admits a self-homeomorphism $\psi$ whose mapping class has infinite order. After perhaps replacing $\psi$ by a finite power of $\psi$, we know that $\psi$ lifts to a self-homeomorphism $\psi_{2}$ of $S$ with the property that, in $\operatorname{Mod}(S)$ we have $\psi_{2} \phi=\phi \psi_{2}$. By replacing $\psi_{2}$ with an appropriate power if necessary, we may assume that $\psi_{2}$ lies in the finite index subgroup $H$.

If $\operatorname{Fix}(\phi)$ is not discrete then $\phi$ is orientation-reversing and $\operatorname{Fix}(\phi)$ is a union of $c>$ 0 simple closed curves. If the quotient $S^{\prime}:=S /\langle\phi\rangle$ has genus $\left(S^{\prime}\right)>0$, then $S^{\prime}$ admits an infinite order self-homeomorphism, which we can then lift as above to obtain $\psi_{2}$. If $\operatorname{genus}\left(S^{\prime}\right)=0$ then $S^{\prime}$ is planar. Picking the outermost curve gives $S^{\prime}$ the structure of a disk with $(c-1)$ open disks removed from its interior. Thus the Euler characteristic 
$\chi\left(S^{\prime}\right)=1-(c-1)=2-c$. Since $S$ is obtained from $S^{\prime}$ by gluing 2 copies of $S^{\prime}$ along its $(\chi=0)$ boundary, we have $2-2 g=\chi(S)=4-2 c$ so that $c=g+1$. Since we are assuming $g>2$, it is clear that $S^{\prime}$ has an infinite order self-homeomorphism, and we are done as above. $\diamond$

\section{Proof of Theorem 1.3}

By Theorem [1.2, $[\operatorname{Isom}(\operatorname{Teich}(S)): \operatorname{Mod}(S)]<\infty$. We pass to the index 2 subgroup Isom $^{+}($Teich $(S))$ of orientation-preserving isometries of Teich $(S)$. Note that that any element $f \in \operatorname{Isom}^{+}(\operatorname{Teich}(S))$ must have Fix $(f)$ of codimension at least 2. Let $f \in \operatorname{Isom}^{+}(\operatorname{Teich}(S))$ with $f \notin \operatorname{Mod}(S)$ be given. Ivanov's theorem on commensurations of $\operatorname{Mod}(S)$ mentioned above implies that the conjugation action of $f$ on some characteristic finite index subgroup $H^{\prime}$ of $\operatorname{Mod}(S)$ agrees on some finite index subgroup $H \leq H^{\prime}$ with conjugation by some element $\phi \in \operatorname{Mod}(S)$. By composing with $\phi$, we may assume the conjugation action of $f$ on $H$ is trivial, i.e. that $f$ centralizes $H$. As $\left[\operatorname{Isom}^{+}(\operatorname{Teich}(S)): H\right]<\infty$, it must be that $f^{n} \in H$ for some $n>1$. Now consider the exact sequence

$$
1 \rightarrow H \rightarrow\langle H, f\rangle \rightarrow\langle f\rangle /\left\langle f^{n}\right\rangle \rightarrow 1
$$

As $H$ is centerless (e.g. since it is finite index in $\operatorname{Mod}(S)$ and so contains a pair of independent pseudo-Anosovs) and since the action of $f$ on $H$ is trivial, it follows that (8) splits, so that $f^{n}=\mathrm{Id}$. By passing to a power of $f$ if necessary, we may assume that $f$ has order some prime $p \geq 2$. Hence $\operatorname{Fix}(f)$ is $\mathbf{Z} / p \mathbf{Z}$-acyclic by Smith theory. Now $\operatorname{Fix}(f)$ has codimension at least 2 , and so has dimension at most 2 . It is also a manifold. Since $\operatorname{dim}(\operatorname{Fix}(f)) \leq 2$, it follows that $\operatorname{Fix}(f)$ is contractible. But, just as noted in Case (B) of Step 3 in $\$ 2$ above, $H$ in these cases is not the fundamental group of a (closed or open) surface; it is also not the fundamental group of a 1-manifold by the same argument. We thus have a contradiction, so that $f$ must be trivial. $\diamond$

\section{References}

[BLM] J. Birman, A. Lubotzky, and J. McCarthy, Abelian and solvable subgroups of the mapping class group, Duke Math. Jour., Vol.50, No.4 (1983), p.1107-1120.

[BKK] M. Bestvina, M. Kapovich and B. Kleiner, Van Kampen's embedding obstruction for discrete groups, Inventiones Math. 150, 219-235 (2002).

[Br] G. Bredon, Introduction to compact transformation groups, Pure and Applied Math., Vol. 46. Academic Press, 1972.

[Bro] K. Brown, Cohomology of Groups, GTM Vol. 87, Springer-Verlag, 1982.

[D] Z. Despotovic, Action dimension of mapping class groups, preprint.

[DH] S. Deng and Z. Hou, The group of isometries of a Finsler space, Pacific J. Math. 207 (2002), no. 1, 149-155. 
[DK] J.J. Duistermaat and J.A.C. Kolk, Lie groups, Universitext. Springer-Verlag, Berlin, 2000 .

[EK] C. Earle and I. Kra, On isometries between Teichmüller spaces, Duke Math. J. 41 (1974), 583-591.

[FW] B. Farb and S. Weinberger, Isometries, rigidity and universal covers, Annals of Math., Vol. 186, No.3, p.915-940 (2008).

[H] J. Hausmann, Homology sphere bordism and Quillen plus construction, in Algebraic K-theory, Evanston1976, Springer Lect. Notes 551, 170-181.

[I1] N. Ivanov, Subgroups of Teichmüller modular groups, translated from the Russian by E. J. F. Primrose and revised by the author, Translations of Math. Monographs, 115, AMS, 1992.

[I2] N.V. Ivanov, Mapping class groups, in Handbook of geometric topology, 523-633, 2002.

[Mc] C. McMullen, The moduli space of Riemann surfaces is Kahler hyperbolic, Annals of Math. (2) 151 (2000), no. 1, 327-357.

[Ro] H.L. Royden, Automorphisms and isometries of Teichmller space, in Advances in the Theory of Riemann Surfaces (Proc. Conf., Stony Brook, N.Y., 1969) pp. 369-383, Ann. of Math. Studies, No. 66., Princeton Univ. Press.

Dept. of Mathematics, University of Chicago

5734 University Ave.

Chicago, Il 60637

E-mail: farb@math.uchicago.edu, shmuel@math.uchicago.edu 